Canadian

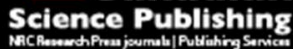

Canadian Geotechnical Journal Revue canadienne de géotechnique

\title{
Identifying local stress increase using a relative apparent stress ratio for populations of mining-induced seismic events
}

\begin{tabular}{|r|l|}
\hline Journal: & Canadian Geotechnical Journal \\
\hline Manuscript ID & cgj-2016-0050.R1 \\
\hline Manuscript Type: & Article \\
\hline Date Submitted by the Author: & 31-May-2016 \\
\hline Complete List of Authors: & $\begin{array}{l}\text { Brown, Laura; Laurentian University; } \\
\text { Hudyma, Martin; Laurentian University }\end{array}$ \\
\hline Keyword: & $\begin{array}{l}\text { Seismicity, Underground Mining, Apparent Stress, Stress Increase, Seismic } \\
\text { Hazard }\end{array}$ \\
\hline & \multicolumn{2}{|l}{} \\
\hline
\end{tabular}

SCHOLARONE ${ }^{m}$

Manuscripts 
Identifying local stress increase using a relative apparent stress ratio for populations of mininginduced seismic events

Author: $\quad$ Laura Brown

Address: $\quad$ Engineering Department

Laurentian University

935 Ramsey Lake Road

Sudbury, ON

P3E 2C6

e-mail: 1g_brown@laurentian.ca

Author: $\quad$ Dr. Martin Hudyma

Address: $\quad$ F-217C, Science Building

Laurentian Univeristy

935 Ramsey Lake Road

Sudbury, ON

P3E 2C6

e-mail: mhudyma@laurentian.ca

Corresponding Author: Laura Brown

Address: 472 St. Raphael St.

Lower Unit

Sudbury, ON

P3B 1M4

e-mail: 1g_brown@laurentian.ca

Phone: $\quad$ (705) $207-3976$ 


\begin{abstract}
Large magnitude mining-induced seismic events can occur as a result of local rock mass stress increase. High apparent stress events can be used as a proxy to identify increases in local rock mass stress conditions. This paper proposes a relative apparent stress ratio (ASR) for quantifying apparent stress of a seismic population. Variations in ASR are analysed over time with reference to seismic hazard inferred from the magnitude of seismic events. The proposed ratio is examined through examples of seismic populations from a single deep Canadian mine.
\end{abstract}

Keywords: Seismicity, Underground Mining, Apparent Stress, Stress Increase, Seismic Hazard. 


\section{Introduction}

Mining-induced seismicity poses a significant risk to the viability of underground mining operations. Seismic events with sufficient energy to damage local excavations are of particular concern. Source parameters enable seismic events to be reviewed quantitatively and provide insight into the local rock mass failure mechanisms driving seismicity. Analysis techniques aim to utilize seismic source parameters to better understand local conditions contributing to rock mass failure.

Rock mass stress is a primary consideration in underground mining. Mine excavations force the in-situ stress within the rock mass to redistribute, generating localized areas of increased and decreased stress. Rock mass failure may occur in the form of large and potentially damaging seismic events when rock mass strength is exceeded by induced stress.

Seismic hazard analysis can be used to differentiate between areas of a rock mass with increased likelihood of producing seismic events of a size that may have an impact on the mining operation. Seismic hazard can also be inferred from local rock mass conditions known to contribute to the occurrence of large events, such as areas of high induced stress or the presence of significant geological structures. Areas of elevated seismic hazard pose an increased risk to the sustainability of the mining operation.

\section{Background}

Seismic hazard is the likelihood of occurrence of a seismic event of a certain size. It varies in space and time within a mine and is probabilistic (Gibowicz and Kijko 1994). In some areas of a mine, the occurrence of large seismic events is more likely, leading to spatial concentrations and variations in large seismic events. Significant changes in excavation geometry can result in large 
local induced stress changes. Large seismic events can occur in association with these geometry and induced stress changes. As a result, seismic hazard varies in space and time within a mine.

Given that there is significant geological uncertainty in a rock mass, it is often difficult to deterministically relate the causal factors associated with rock mass failure with the occurrence of a particular event. In many circumstances, there is a population of hundreds or thousands of recordable seismic events associated with a rock mass failure process, often occurring over a time period of weeks or months. These events provide insight into when and approximately where the rock mass is failing.

Andrieux et al. (2008) found that the seismic response to mining is strongly related to the stressstrain response of the confined rock mass. They report that large seismic events are more likely to occur immediately after mining-induced stresses exceed the peak strength of the rock mass. Large seismic events may also be more frequent as the rock mass exhibits considerable post peak softening. Monitoring mining induced stress changes therefore provides significant insight into the likely seismic response.

The seismic source parameter apparent stress is a model independent measure of stress release at a seismic source (Gibowicz et al. 1990, 1991). Proposed by Wyss and Brune (1968), apparent stress was defined as:

$$
\sigma_{\mathrm{a}}=\mu(\mathrm{E} / \mathrm{Mo})
$$

where,

$$
\begin{aligned}
& \sigma_{\mathrm{a}}=\text { Apparent Stress }(\mathrm{Pa}) \\
& \mu=\text { Shear Modulus of Rigidity of the Source Material }\left(\mathrm{N} / \mathrm{m}^{2}\right)
\end{aligned}
$$




$$
\begin{aligned}
& \mathrm{E}=\operatorname{Seismic} \text { Energy }(\mathrm{J}) \\
& \mathrm{Mo}=\text { Seismic Moment }(\mathrm{Nm})
\end{aligned}
$$

\section{Apparent Stress Recorded in Underground Mines}

A logarithmic relation can be identified between radiated seismic energy and moment in a wellrecorded seismic population of sufficient size. Figure 1 is an energy-moment relation for a large seismic population at a deep Canadian Mine. Radiated energy increases proportionally to the seismic moment. The average relation for the events shown in Figure 1 can be expressed as $\log (E)=1.18 \times \log (M)-6.99$. Seismic energy can vary by approximately one order of magnitude from the relation for a given value of seismic moment.

Events in Figure 1 are also coloured and sized to apparent stress. Events with the largest radiated seismic energy for a given moment represent the highest apparent stress events. Events with relatively large energy and moment values, towards to upper right of the relation, also represent high apparent stress events.

Event magnitude is used as a measure of size or intensity of a seismic event. Magnitude is closely related to the radiated seismic energy. Events that radiate increased quantities of energy typically possess larger magnitudes. Richter (1935) proposed a logarithmic magnitude scale that is widely used. This paper presents event size as an equivalent Richter magnitude (MR) calibrated from a consistent local magnitude scale.

Seismic populations associated with elevated seismic hazard possess the potential to produce seismic events capable of having adverse effects on the mining operation. Butler (1997) 
identified seismic events MR $>1$ as large and potentially damaging. This paper will use the same convention, Richter magnitude $>+1$ events $(\mathrm{MR}>1)$ will be referred to as large magnitude events. In a study of several rockburst prone mines, Morissette (2015) found that approximately $4 \%$ of events MR $>1$ resulted in rockburst damage. Of these events, 33\% were major rockbursts, causing more than 100 tonnes of rock to be displaced.

Figure 2 is a presentation of the data in Figure 1 with events colored and sized to equivalent Richter magnitude. Dotted lines correspond to apparent stress thresholds shown in Figure 1. Large magnitude events $(\mathrm{MR}>1)$ predominantly have high apparent stress values (Figure 2). A threshold of $450 \mathrm{kPa}$ is used to define high apparent stress, as less than $1 \%$ of the total population of events have an apparent stress of greater than $450 \mathrm{kPa}$. Less than $0.8 \%$ of small magnitude events $(\mathrm{MR}<1)$ shown in Figure 2 possess high apparent stress.

The frequency of occurrence and the magnitude of seismic events follows a power law relation (Gutenberg and Richter 1944). With each order of increase in magnitude, the quantity of events decreases by a factor of 10 . Figure 3 is a series of cumulative distributions of apparent stress for the seismic events shown in Figure 2. The change in apparent stress between increasing magnitude populations in Figure 3 indicates a positive relation between event magnitude and apparent stress. As magnitude, or event size, increases, apparent stress increases. Seismic analysis techniques that primarily consider high apparent stress are therefore considering large Various works have shown a relation between apparent stress and increasing stress conditions in mines (Mendecki et al. 1999; Simser et al. 2003; Hudyma 2008). In-situ rock mass stress increases with depth and is reflected in the apparent stress of seismic populations associated with different mining levels. Figure 4 is a series of cumulative distributions of apparent stress for levels of increasing depth at a deep Canadian Mine. An elevation change of approximately 560 
m exists between the upper and lower level shown. In Figure 4, the increased depth of each subsequent mining level is reflected in the significant increase in associated apparent stress. Apparent stress is a relative measure of stress release at a seismic source. In this case, the stress release is shown to increase with depth, as median apparent stress recorded increases by approximately $125 \%$ from the 203 level to the 262 level.

Geological and stress driven variations in the local rock mass can also have a significant influence on the relative levels of recorded apparent stress. For example, Figure 5 contains two cumulative distributions of apparent stress for two different mines. Variations in the local mining environments, quantified by uniaxial compressive strength (UCS) of the rock mass and mining depth, are reflected in the significant difference in apparent stress distributions.

\section{Apparent Stress Ratio (ASR)}

From Figures 4 and Figure 5, high apparent stress depends strongly on local site conditions. One means of identifying anomalous apparent stress levels within a population is through a relative ratio. Apparent stress ratio (ASR) is proposed as a means of quantifying local apparent stress relative to itself. For any given seismic population ASR is calculated as the ratio of the $80^{\text {th }}$ percentile to the $20^{\text {th }}$ percentile for a cumulative distribution of apparent stress:

$$
\operatorname{ASR}=\sigma_{\mathrm{a} 80} / \sigma_{\mathrm{a} 20}
$$

where:

$$
\begin{aligned}
& \sigma_{\mathrm{a} 80}=\text { apparent stress } 80 \text { th percentile. } \\
& \sigma_{\mathrm{a} 20}=\text { apparent stress } 20 \text { th percentile. }
\end{aligned}
$$

From a comprehensive study of 50 seismic populations at a deep Canadian mine (Brown 2015), for a given population the $80^{\text {th }}$ and $20^{\text {th }}$ percentile values approximate the beginning and end of 
the linear relation of the dataset, as shown in Figure 6 . The $80^{\text {th }}$ and $20^{\text {th }}$ percentile values allow for the majority of an apparent stress distribution to be considered while ignoring the influence of the extremities of the population. The advantage of using a relative apparent stress ratio to identify anomalously high apparent stress is that the ratio accounts for local variations in rock mass strength and mining depth.

The $80^{\text {th }}$ and $20^{\text {th }}$ percentiles for the population shown in Figure 6 are approximately $95 \mathrm{kPa}$ and $16 \mathrm{kPa}$ respectively. The corresponding ASR is 5.8. The detailed study (Brown 2015) found that an ASR of greater than 3.0 was indicative of areas of moderate to high induced stress for this mine. As a generalization, these areas of the mine also tend to be more prone to the occurrence of large seismic events.

\section{Variations in ASR}

Relating increases and decreases in ASR over time to mining activities works towards an understanding of the temporal seismic response to mining. At any location in a mine, the occurrence of seismic events is usually related to recent mining activities. While investigating the seismic response to mining in South African mines, van Aswegan (2005) noted that mine planning cycles considered mine extraction over a period of a few months. He referred to this as a medium term time frame. van Aswegan (2005) referred to long term as a sufficient time to incorporate major mine planning changes. In the context of a deep Canadian bulk mining operation, this would be several months or more. It is proposed to investigate increasing and decreasing trends in ASR over similar time periods. Medium term seismic assessment will refer to seismicity recorded in the preceding three months. Long term seismic assessment will refer to seismicity recorded in the preceding year. 
Due to the limit of completeness of the seismic dataset in use, only events greater than or equal to Richter magnitude -0.5 will be considered. All events with magnitudes above this bound have been reliably recorded by the seismic monitoring system in all parts of the sensor array. Events smaller than this bound are not always recorded reliably, and consequently, will not be considered in this paper.

To investigate temporal variations in apparent stress and ASR, a time history chart will be used. Events are plotted according to date and local magnitude on the $\mathrm{x}$ and $\mathrm{y}$-axis respectively. ASR is represented by a line plotted on the secondary y-axis. Events are colored and sized to apparent stress to better demonstrate trends in ASR over time. Figure 7 is an Apparent Stress Ratio Time History chart for a seismic population containing numerous seismic events located within a $30 \mathrm{~m}$ radius sphere, including two large magnitude events $(M R>1)$. Individual ASR values are calculated using long term seismic assessment - considering events within a one year preceding time period.

The seismic population shown in Figure 7 is characterized by two periods of intense seismic activity, separated by a period of inactivity. In 2006, small magnitude events begin to occur with low to moderate apparent stress. ASR values remain relatively stable at values indicative of low to moderate stress increase $(1<\mathrm{ASR}<3)$ (Brown 2015). A period of inactivity, beginning in 2007, is abruptly ended in July 2007, with the occurrence of a high apparent stress (375 $\mathrm{kPa})$ event, suggesting a local stress increase. The one year trailing ASR values gradually increases in early to mid-2007, reaching a value of 6 prior to the large event in July 2007.

An ASR of 6, highly indicative of stress increase, precedes the subsequent large magnitude (MR $=1.5)$ and high apparent stress (1 $246 \mathrm{kPa})$ event. The apparent stress of subsequent events is significantly lower (AS $<100 \mathrm{kPa}$ ) and may be a reflection of a local stress decrease. 
Large variations in the apparent stress of events in the remainder of 2007 and 2008 produce ASR values indicative of high stress conditions (ASR > 6). A second large event occurs in early 2009 $(M R=2.4)$. Although this event is subsequent to a period of inactivity, long term seismic assessment enables ASR to reflect the increased apparent stress $80^{\text {th }}$ percentile over the preceding year. An ASR of 10, highly indicative of stress increase, precedes the large event.

Increases in ASR are usually a direct result of an increase in the $80^{\text {th }}$ apparent stress percentile used in calculation. In Figure 8, events are plotted according to date and apparent stress on the $\mathrm{x}$ and $y$-axis, respectively. The $20^{\text {th }}$ and $80^{\text {th }}$ apparent stress percentiles are ploted by a line on the primary y-axis. ASR is represented by a line plotted on the secondary y-axis. Events are coloured and sized to equivalent Richter magnitude. Individual apparent stress percentiles and ASR values are calculated using a one year trailing seismic population. The $20^{\text {th }}$ apparent stress percentile remains relatively stable, at approximately $20 \mathrm{kPa}$, throughout the population life. Increases in ASR directly correspond to increases in the $80^{\text {th }}$ apparent stress percentile. As the degree of separation between the $80^{\text {th }}$ and $20^{\text {th }}$ percentiles increases, ASR increases. Thus, the increasing ASR is driven primarily by the addition of high apparent stress events to the cumulative apparent stress distribution.

Medium term seismic assessment is more representative for areas within a rock mass that experience large stress changes in relatively short periods of time - over a few months. Figure 9 is an Apparent Stress Ratio Time History chart for a seismic population containing a large magnitude event $(M R>1)$. All events shown occur within a $30 \mathrm{~m}$ radius sphere. Individual ASR values are calculated using medium term seismic assessment - considering events within a three month preceding time period. 
The seismic population shown in Figure 9 is characterized by a relatively low event rate and a single large magnitude event. In March-April 2013, an increase in event occurrence begins to generate ASR values greater than 3, indicative of moderate to high stress increase. The subsequent event is a large magnitude $(M R=2.2)$ and high apparent stress $(2271 \mathrm{kPa})$ event, occurring in Aug 2013. Event rate remains relatively constant following the large event and after Jan 2014, ASR values drop to levels indicative of low stress (ASR < 2). No further large events are recorded.

Seismic populations shown in Figures 7 and 9, were representative of areas within the rock mass that experienced a local stress increase contributing to the occurrence of large events. This was reflected in ASR through the increasing apparent stress $80^{\text {th }}$ percentile of the population.

Areas in a mine experience varying levels of induced stress over time, which can be reflected in variations in ASR over time. Figure 10 is an Apparent Stress Ratio Time History chart for a seismic population containing two large magnitude events $(M R>1)$. All events shown occur within a $30 \mathrm{~m}$ radius sphere. Individual ASR values are calculated using long term seismic assessment - considering events within a one year preceding time period. The seismic population shown in Figure 10 contains a period of stress increase (late 2008 to early 2009), followed by a period of decreased stress conditions (late 2009, 2010 and 2011). Events occurring in 2008 have predominately low apparent stress values. As larger apparent stress events are introduced to the population in the end of 2008, ASR values become indicative of moderate to high stress increase (ASR > 3). In the beginning of 2009, all events exhibit relatively high apparent stress, leading to large increases in ASR (ASR > 10), indicative of high stress increase. A large magnitude $(\mathrm{MR}=$ 2.2) event occurs in Oct 2009, preceded by an ASR of 13. 
In late 2009, the trailing one year time window used to calculate ASR no longer considers the relatively low apparent stress values in the beginning of the population life. As a result, ASR values return to levels indicative of low to moderate stress increase $(2<\mathrm{ASR}<4)$. A large magnitude $(\mathrm{MR}=1.3)$ but low apparent stress $(94 \mathrm{kPa})$ event occurs in July 2010. Following this event, ASR levels remain stable at levels indicative of low stress (ASR < 2). Little variation exists in the apparent stress of events occurring in 2011 and no further large events occur within the population. This example demonstrates that ASR is capable of reflecting both stress increase and stress decreases within a rock mass.

In Figure 10, variations in the local induced stresses over time were reflected in large scale increases and decreases in ASR. Figure 11 is an Apparent Stress Ratio Time History chart for a seismic population containing sequences of numerous large magnitude events $(\mathrm{MR}>1)$. The large magnitude event sequences in this population may be stress driven failure due to spatial proximity within a sill pillar with high induced stress. Individual ASR values are calculated using long term seismic assessment - considering events within a one year preceding time period. The seismic population shown in Figure 11 contains three distinct large event sequences occurring in mid-2009, mid-2010 and mid-2011. A significant portion of large magnitude events (MR $>1)$ occur during and independent of event sequences. ASR is relatively constant at values greater than 3, indicative of moderate to high stress, throughout the life of the population. Large events may not by a result of local stress increase, but rather the continued high stress conditions within the local sill pillar. ASR remains relatively constant as there are both low and high apparent stress events occurring over time. This example demonstrates that ASR is capable of reflecting continued high stress conditions within a rock mass.

\section{Peak ASR}


The historic seismic response is an indicator of the potential future seismic response. The presence of a high ASR time period within a seismic population can be indicative of future high ASR and possibly the occurrence of large magnitude events. Peak ASR is defined as the largest ASR value reached by a seismic population. Individual ASR values are calculated using a moving time window but the peak ASR value is a worst-case indicator, not decreasing over time. The peak value serves as a measure of the largest stress change that has occurred in the seismic event population. Figure 12 is an Apparent Stress Ratio Time History chart for a seismic population containing two large magnitude events $(M R>1)$. All events shown occur within a 30 $\mathrm{m}$ radius sphere. Individual ASR values are calculated using long term seismic assessment considering events within a one year preceding time period.

With the occurence of each subsequent large magnitude event in Figure 12, peak ASR increases. Prior to the first large event $(\mathrm{MR}=1.6)$, the largest ASR value reached by the population is approximately 7 . The influence of the large event on the apparent stress distribution generates an increased ASR of 10. This peak is then exceeded by an ASR value of 11, preceeding the second large event $(\mathrm{MR}=1.8)$. The influence of the second large event further increases peak ASR to 12. An overall peak ASR of 12 for this seismic population indicates a ratio of $12: 1$ for the $80^{\text {th }}$ to $20^{\text {th }}$ apparent stress percentile during a one year time period. The peak ASR indicates a large scale stress increase and may be a reflection of the ability of the local rock mass to concentrate stress.

A recent study (Brown 2015), found time periods of high peak ASR to be well correlated to the occurrence of large magnitude events $(M R>1)$. Figure 13 depicts the cumulative distributions of peak ASR for 25 spatially separate control populations (all seismic events MR $<1$ ) and 25 spatially separate test populations (containing seismic events MR > 1). Individual ASR values 
are calculated using medium term seismic assessment - considering events within a three month proceeding time period.

In Figure 13, test populations (containing large magnitude events), possess significantly larger peak ASR values. This is an indication that seismic populations which have produced large magnitude events typically experience time periods of larger stress increase relative to populations with smaller magnitude events. Approximately $90 \%$ of test populations possess a peak ASR greater than 3, which is indicative of moderate to high stress increase.

\section{Discussion}

ASR is an empirical analysis technique. It must be calibrated and tested prior to site application. The lower bound of MR $=-0.5$ used in this analysis eliminates the influence of smaller magnitude events. Large magnitude events $(M R>1)$ are not necessarily driven by the same failure processes as smaller magnitude events (Hudyma 2008). Due to the power law relation between event frequency and magnitude, the inclusion of smaller magnitude events will skew the cumulative apparent stress distribution used in ASR calculation. This will decrease the $20^{\text {th }}$ apparent stress percentile generating high ASR values. It is recommended a lower bound of MR $=-0.5$ be maintained in the application of ASR to alternative seismic data sets.

The use of varying seismic assessment time periods enables ASR to reflect varying stress conditions related to varying scales of mining activities. The most appropriate assessment time period is dependent on the local rock mass response to mining. Medium term is more suited for regions of a rock mass that are capable of experiencing significant stress increase over time periods in the range of weeks to months. More gradual stress change is better represented by 
long term assessment. Understanding the impact of the assessment time period on the calculation of ASR values is essential for inferring seismic hazard.

\section{Conclusions}

Anomalously high apparent stress recorded in seismic events occurs as a result of increasing stress conditions in a rock mass. ASR reflects the presence of high apparent stress events in a seismic population. These events are typically larger magnitude events corresponding to potential rock mass instability and increased seismic hazard.

Increasing stress conditions may contribute to the likelihood of occurrence of large seismic events. The relative stress increase associated with a seismic population can be quantified using ASR. Peak ASR is a reflection of the largest stress change experienced by a seismic population and provides a means of assessing potential for stress related rock mass instability. A detailed back analysis in a deep Canadian mine concluded large ASR values correlate well to areas of elevated seismic hazard (Brown 2015).

Defining high apparent stress is challenging due to variations in local rock mass stress and geological conditions. ASR eliminates the requirement for arbitrary threshold values. However, local experience is still needed to identify high ASR conditions. In this paper, ASR values greater than 3 were indicative of moderate to high stress increase. These values are consistent with a broader study of several years of seismic data at the same deep Canadian Mine (Brown 2015). 


\section{Acknowledgments}

This research is supported by Agnico Eagle's LaRonde Mine, the Goodman School of Mines, the

Natural Sciences and Engineering Research Council, and the Canadian Engineering Memorial

Foundation. 


\section{References}

Andrieux, P.P., Hudyma, M.R., O’Connor, C.P., Li, H., Cotesta, L., and Brummer, R.L. 2008. Calibration of large-scale three-dimensional non-linear numerical models of underground mines using microseismic data. In Proceedings of the First International FLAC/DEM Symposium, Minneapolis, 25-27 August 2008.

Brown, L.G. 2015. Seismic hazard evaluation using apparent stress ratio for mining-induced seismic events. M.A.Sc. thesis, Department of Engineering, Laurentian University, Sudbury, O.N.

Butler, A.G. 1997. Space-time clustering of potentially damaging seismic events and seismic viscosity in Western Deep Levels East and West Mines. In Proceedings of the Fourth International Symposium on Rockbursts and Seismicity in Mines, Krakow, Poland, 11-14 August 1997. pp. 89-93.

Gibowicz, S.J. and Kijko, A. 1994. An introduction to mining seismology. Academic Press, San Diego.

Gibowicz, S.J., Harjes, H.-P., and Schafer. M. 1990. Source parameters of seismic events at Heinrich Robert mine, Ruhr basin, federal republic of Germany: evidence for nondouble-couple events. Bulletin of the Seismological Society of America, 80(1): 88-109.

Gibowicz, S.J., Young. R.P., Talebi. S., and Rawlence, D.J. 1991. Source parameters of seismic events at the underground research laboratory in Manitoba, Canada: scaling relation for events with moment magnitude smaller than -2. Bulletin of the Seismological Society of America, 81(4): 1157-1182. 
Gutenberg, B., and Richter, C.F. 1944. Frequency of earthquakes in California. Bulletin of the Seismological Society of America, 34(4): 185-188.

Hudyma, M.R. 2008. Analysis and interpretation of clusters of seismic events in mines. Ph.D. thesis, Department of Civil and Resource Engineering, The University of Western Australia, Perth, W.A.

Mendecki, A.J., van Aswegen, G., and Mountfort, P. 1999. A guide to routine seismic monitoring in mines. A Handbook on Rock Engineering Practices for Tabular Hard Rock Mines. Creda Communications, Cape Town.

Morissette, P.N.R. 2015. A ground support design strategy for deep underground mines subjected to dynamic-loading conditions. Ph.D. thesis, Department of Civil Engineering, The University of Toronto, Toronto, O.N.

Richter, C.F. 1935. An instrumental earthquake magnitude scale. Bulletin of the Seismological Society of America, 25(1): 1-32.

Simser, B.P., Falmagne, V., Gaudreau, D., and MacDonald, T. 2003. Seismic response to mining at the Brunswick mine. Canadian Institute of Mining and Metallurgy Annual General Meeting, Montreal.

van Aswegen, G. 2005. Routine seismic hazard assessment in some South African mines. In Proceedings of the Sixth International Symposium on Rockbursts and Seismicity in Mines, Perth, Western Australia, 9-11 March 2005. Australian Centre of Geomechanics, Perth, pp. 437-444.

Wyss, M. and Brune, J.N. 1968. Seismic moment, stress and source dimensions for earthquakes in the California-Nevada region. Journal of Geophysical Research, 73(14): 4681-4694. 


\section{Figure Captions}

Figure 1: Energy-Moment Relation chart for a large seismic population at a deep Canadian Mine. A relation is shown for which seismic energy can vary by approximately one order of magnitude for a given value of seismic moment. Large apparent stress events are predominantly concentrated at large radiated seismic energy values for given values of seismic moment.

Figure 2: Energy-Moment Relation chart for a large seismic population at a deep Canadian Mine. A relation is shown for which seismic energy can vary by approximately one order of magnitude for a given value of seismic moment. Large magnitude events are predominantly concentrated at large radiated seismic energy and seismic moment values which also represent high apparent stress. Dotted lines refer to apparent stress thresholds shown in Figure 1.

Figure 3: Cumulative distributions of apparent stress for a large seismic population at a deep Canadian mine. Events are separated into populations by Richter magnitude. Significant changes in apparent stress of different magnitude ranges indicates apparent stress increases as event magnitude increases.

Figure 4: Cumulative distributions of apparent stress for a series of levels at a deep Canadian Mine (modified from Brown 2015). Level values refer to approximate depth below surface (203 Level is approximately $2030 \mathrm{~m}$ below surface). Significant changes in apparent stress of different mining levels indicates apparent stress increases as depth of mining increases.

Figure 5: Cumulative distributions of apparent stress for a mine representative of high UCS (220 $\mathrm{MPa}$ ) and mining at depths greater than $1500 \mathrm{~m}$ and a mine representative of lower UCS (130 $\mathrm{MPa}$ ) and mining at depths less than $800 \mathrm{~m}$. Variations in the local mining environments are reflected in the significant difference in apparent stress distributions. 
Figure 6: Cumulative distribution of apparent stress for a seismic population at a deep Canadian Mine. All events shown occur within a $30 \mathrm{~m}$ radius sphere over a time span of approximately five years. Black dashed lines approximate the extent of the linear relation for the data, corresponding to the $20^{\text {th }}$ and $80^{\text {th }}$ apparent stress percentiles.

Figure 7: Apparent Stress Ratio Time History chart for a seismic population containing large magnitude events $(\mathrm{MR}>1)$. All events shown occur within a $30 \mathrm{~m}$ radius sphere. Individual ASR values are calculated for long term seismic assessment - considering events within a one year proceeding time period. ASR increases prior to the occurrence of large magnitude seismic events.

Figure 8: Apparent Stress Percentile Time History chart for a seismic population containing large magnitude events $(\mathrm{MR}>1)$. All events shown occur within a $30 \mathrm{~m}$ radius sphere. Individual apparent stress percentile and ASR values are calculated using a 1 year trailing seismic population - considering events within a one year preceding time period. Apparent stress $20^{\text {th }}$ percentile values remain relatively stable over time with increases in ASR being driven by increases in the apparent stress $80^{\text {th }}$ percentile values.

Figure 9: Apparent Stress Ratio Time History chart for a seismic population containing large magnitude events $(\mathrm{MR}>1)$. All events shown occur within a $30 \mathrm{~m}$ radius sphere. Individual ASR values are calculated for medium term seismic assessment - considering events within a three month preceding time period. ASR increases prior to the occurrence of the large magnitude seismic event.

Figure 10: Apparent Stress Ratio Time History chart for a seismic population containing large magnitude events $(\mathrm{MR}>1)$. All events shown occur within a $30 \mathrm{~m}$ radius sphere. Individual 
ASR values are calculated for long term seismic assessment - considering events within a one year preceding time period. ASR values reflect both a local increase and decrease in rock mass stress conditions.

Figure 11: Apparent Stress Ratio Time History chart for a seismic population containing sequences of large magnitude events $(M R>1)$. Individual ASR values are calculated for long term seismic assessment - considering events within a one year preceding time period. ASR values are indicative of moderate to high stress conditions throughout the population life, reflecting both stress increases and continued high rock mass stress conditions.

Figure 12: Apparent Stress Ratio Time History chart for a seismic population containing large magnitude events $(\mathrm{MR}>1)$. All events shown occur within a $30 \mathrm{~m}$ radius sphere. Individual ASR values are calculated for long term seismic assessment - considering events within a one year preceding time period. Peak ASR values continue to increase over time as larger magnitude events are introduced to the population.

Figure 13: Cumulative distribution of peak ASR values for control and test populations calculated using medium term seismic assessment - considering events within a three month preceding time period (modified from Brown 2015). Test populations, containing large magnitude events, possess significantly larger peak ASR values. 


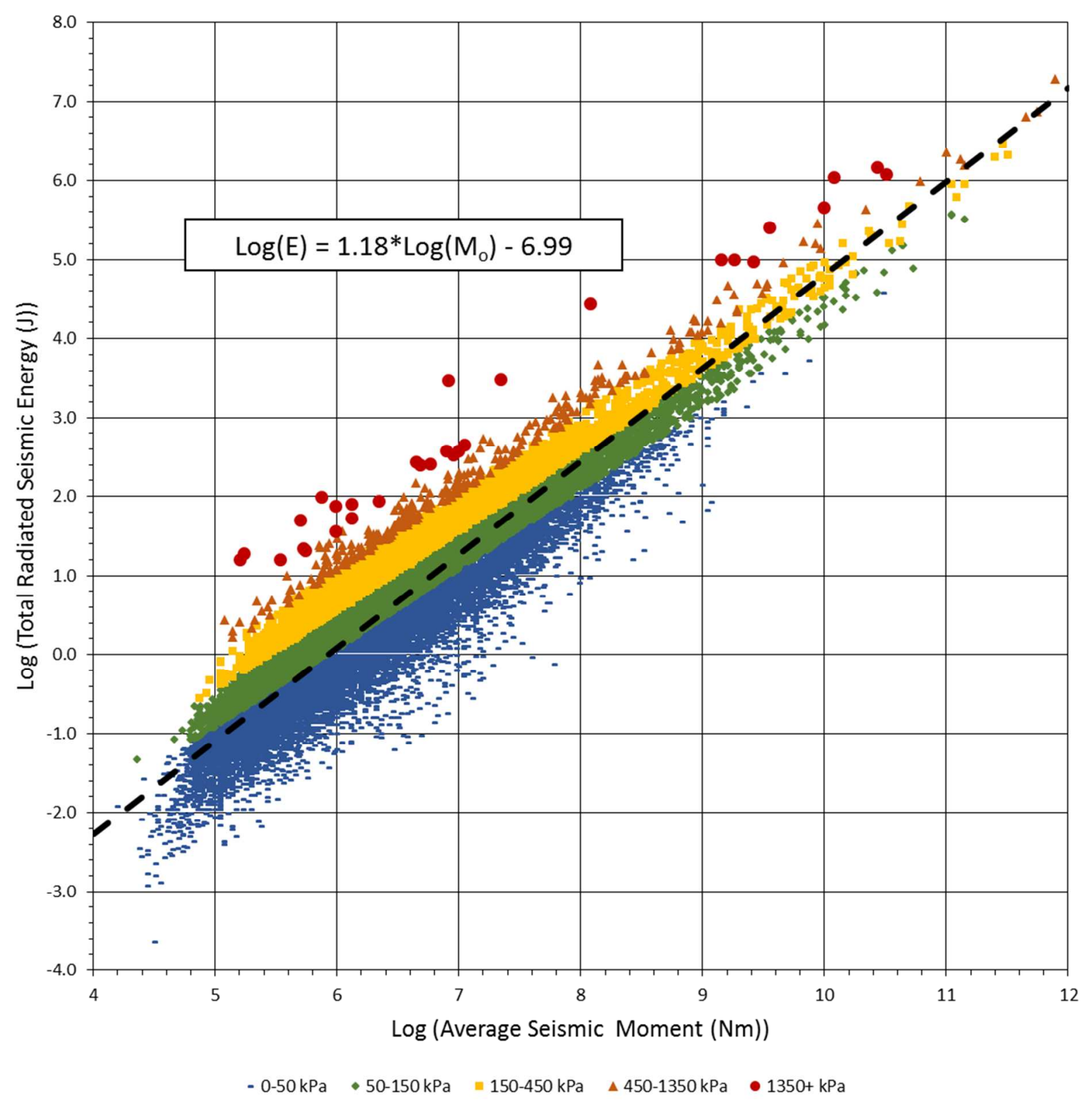

Figure 1: Energy-Moment Relation chart for a large seismic population at a deep Canadian Mine. A relation is shown for which seismic energy can vary by approximately one order of magnitude for a given value of seismic moment. Large apparent stress events are predominantly concentrated at large radiated seismic energy values for given values of seismic moment. 


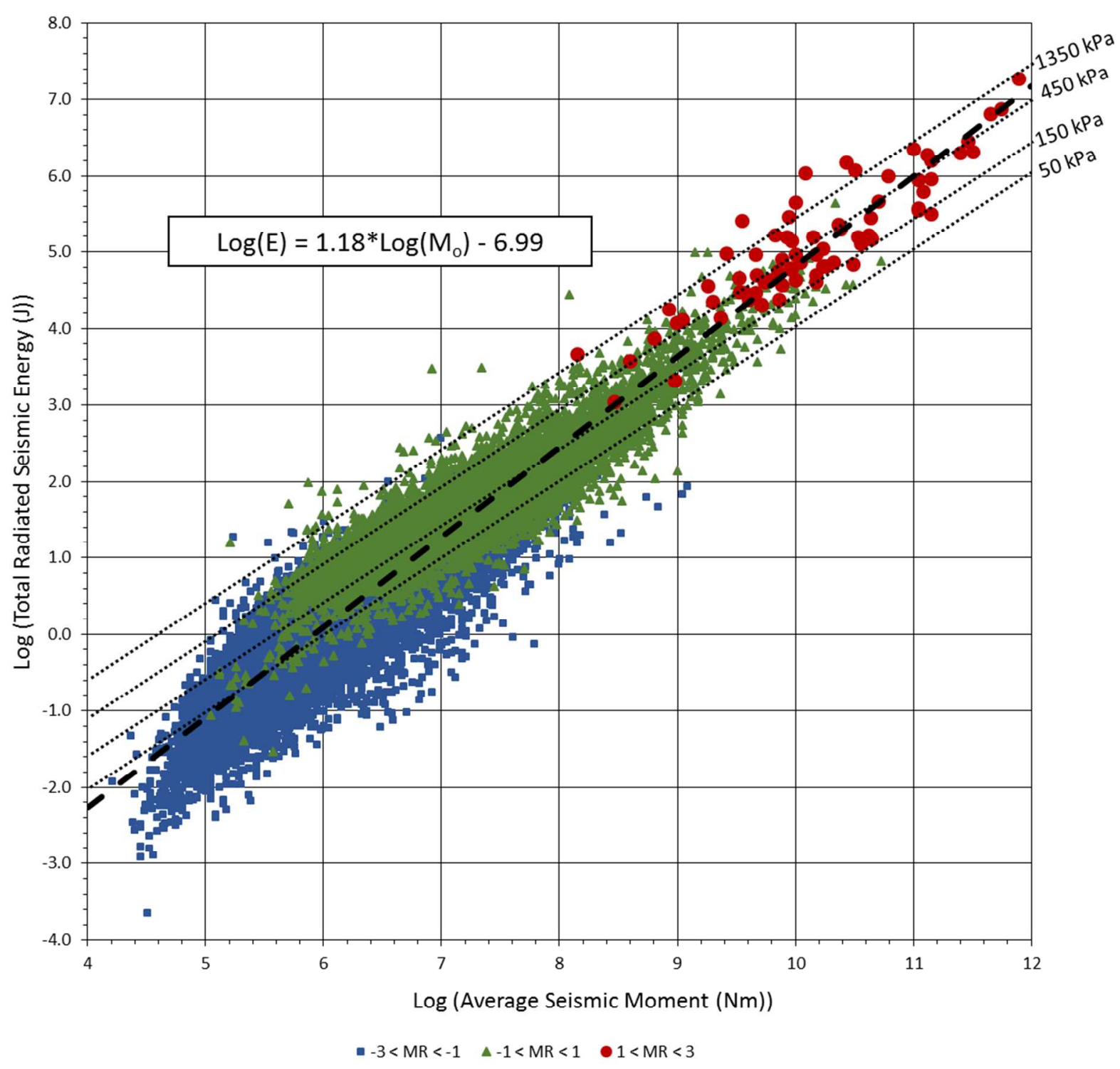

Figure 2: Energy-Moment Relation chart for a large seismic population at a deep Canadian Mine. A relation is shown for which seismic energy can vary by approximately one order of magnitude for a given value of seismic moment. Large magnitude events are predominantly concentrated at large radiated seismic energy and seismic moment values which also represent high apparent stress. Dotted lines refer to apparent stress thresholds shown in Figure 1. 


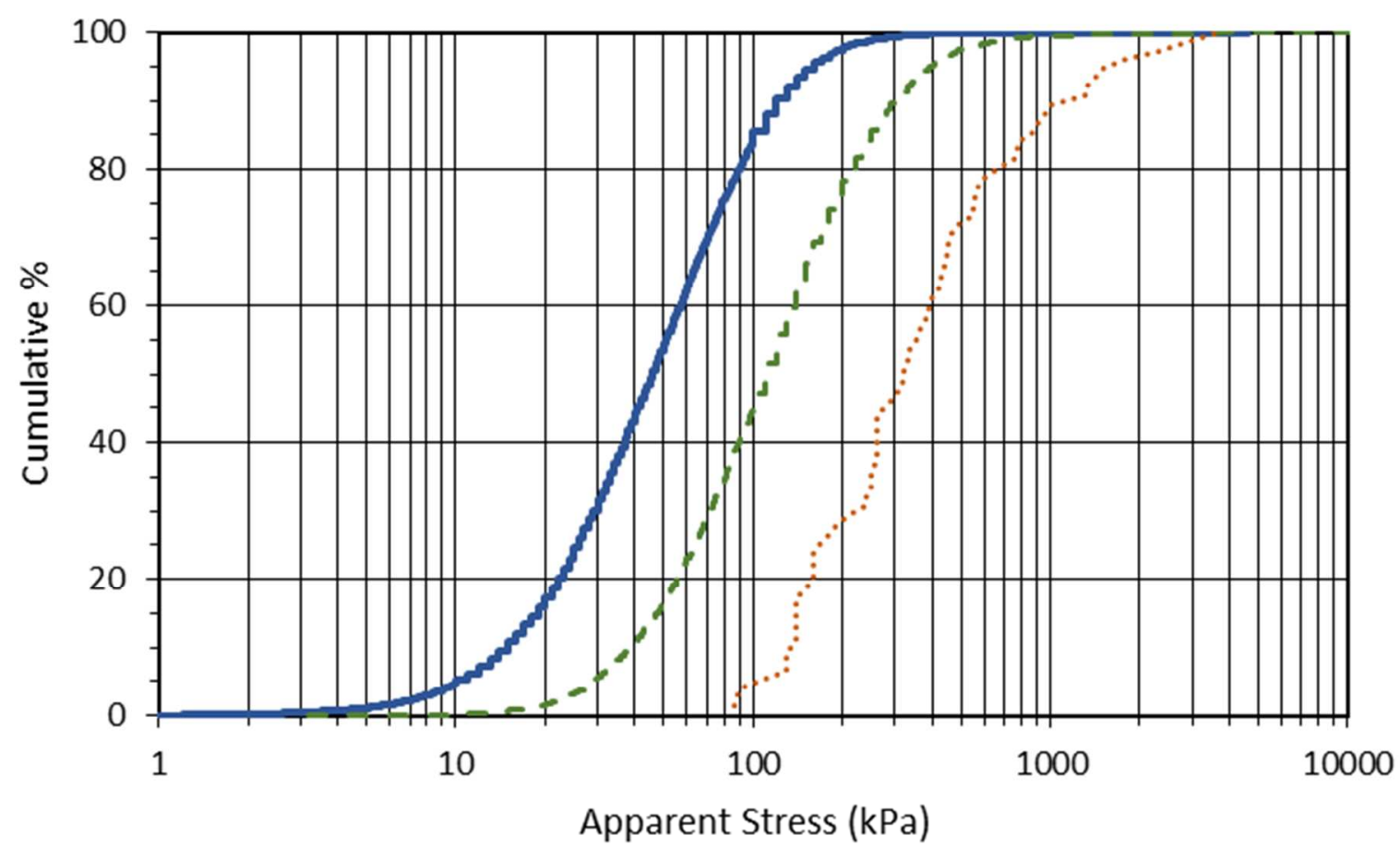

$-3<\mathrm{MR}<-1 \quad----1<\mathrm{MR}<1$

$1<\mathrm{MR}<3$

Figure 3: Cumulative distributions of apparent stress for a large seismic population at a deep Canadian mine. Events are separated into populations by Richter magnitude. Significant changes in apparent stress of different magnitude ranges indicates apparent stress increases as event magnitude increases. 


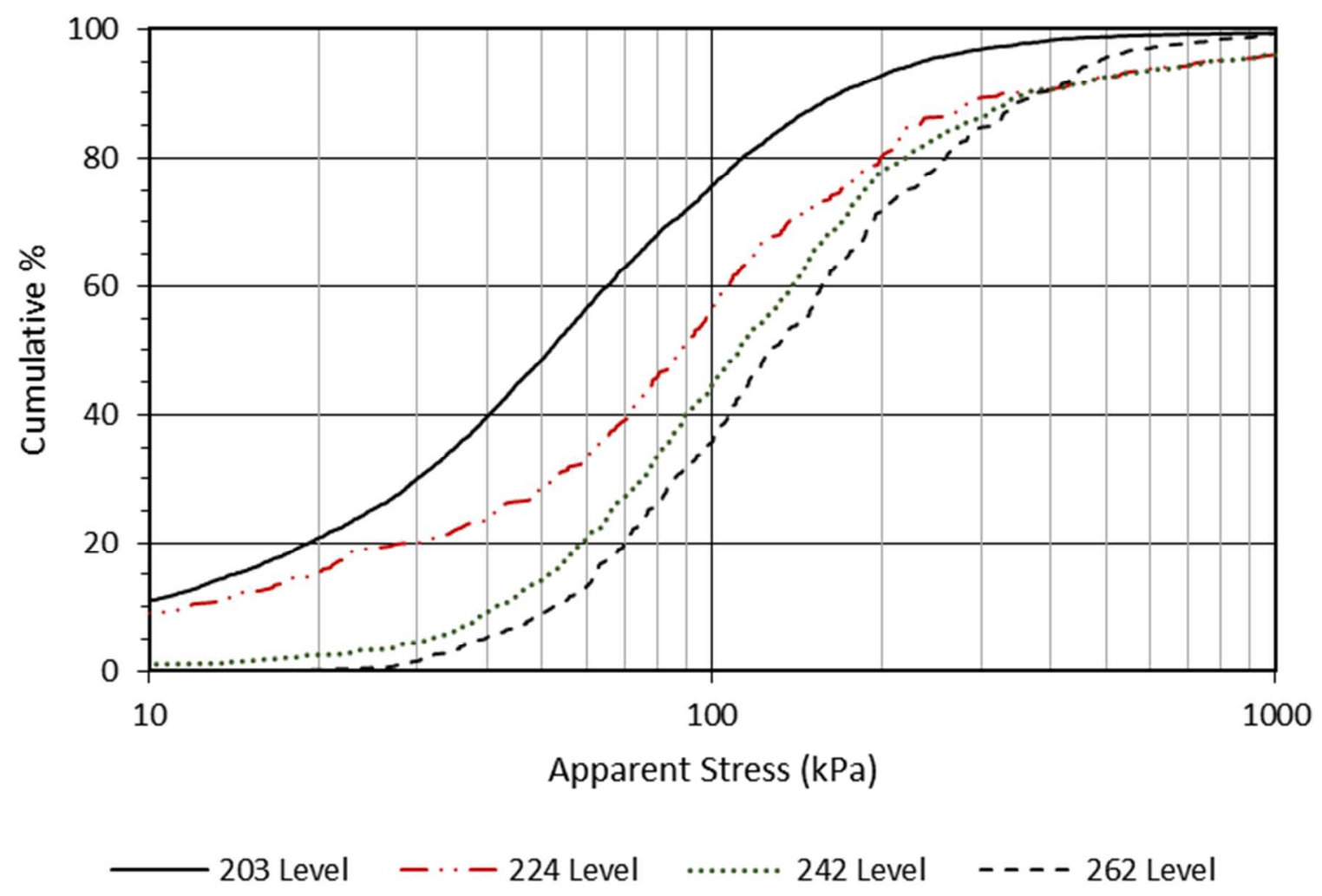

Figure 4: Cumulative distributions of apparent stress for a series of levels at a deep Canadian Mine (modified from Brown 2015). Level values refer to approximate depth below surface (203 Level is approximately $2030 \mathrm{~m}$ below surface). Significant changes in apparent stress of different mining levels indicates apparent stress increases as depth of mining increases. 


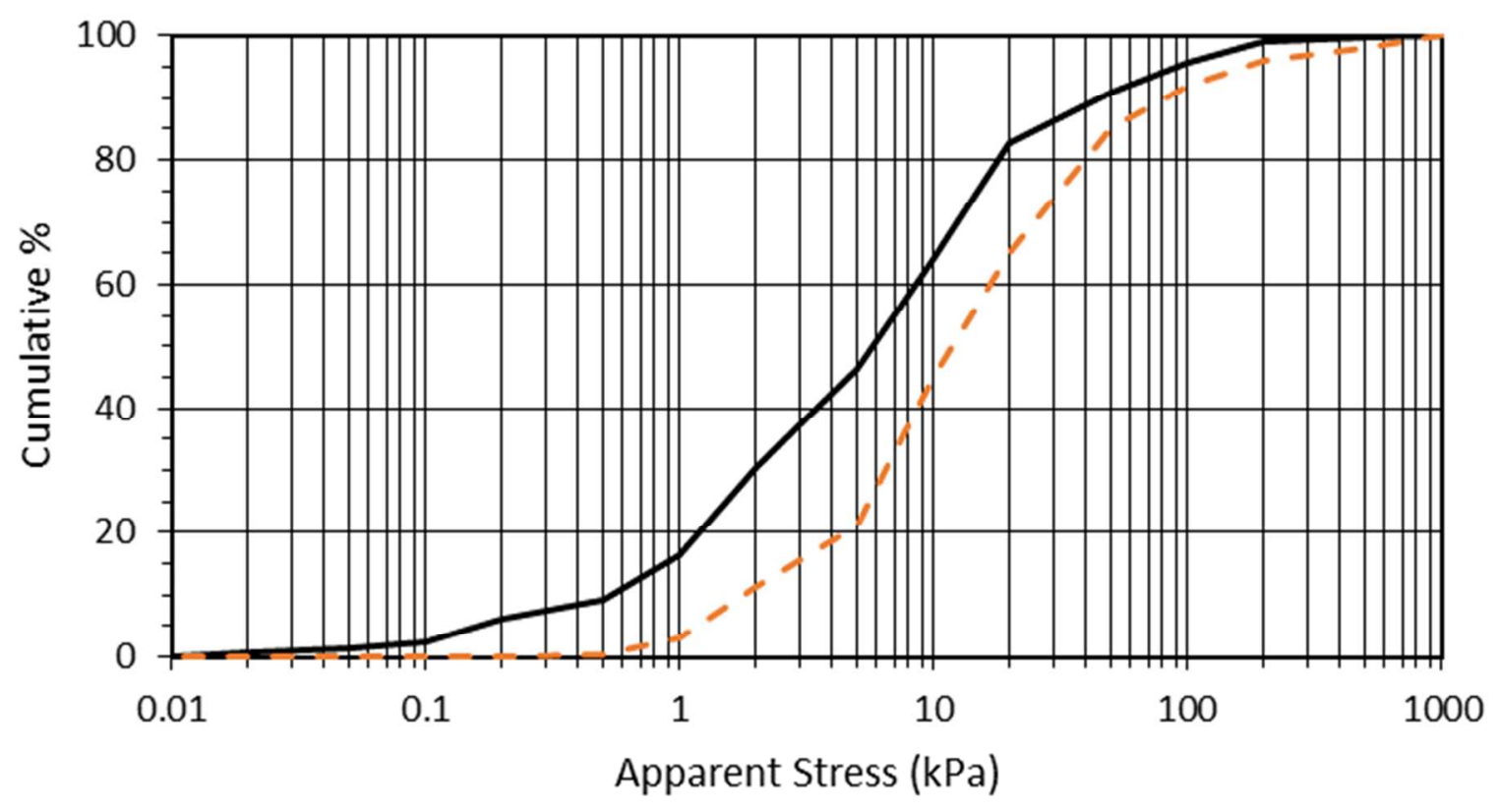

— Low UCS \& Depth $<800 \mathrm{~m} \quad-\quad-\quad$ High UCS \& Depth $>1500 \mathrm{~m}$

Figure 5: Cumulative distributions of apparent stress for a mine representative of high UCS (220 $\mathrm{MPa}$ ) and mining at depths greater than $1500 \mathrm{~m}$ and a mine representative of lower UCS (130 $\mathrm{MPa}$ ) and mining at depths less than $800 \mathrm{~m}$. Variations in the local mining environments are reflected in the significant difference in apparent stress distributions. 


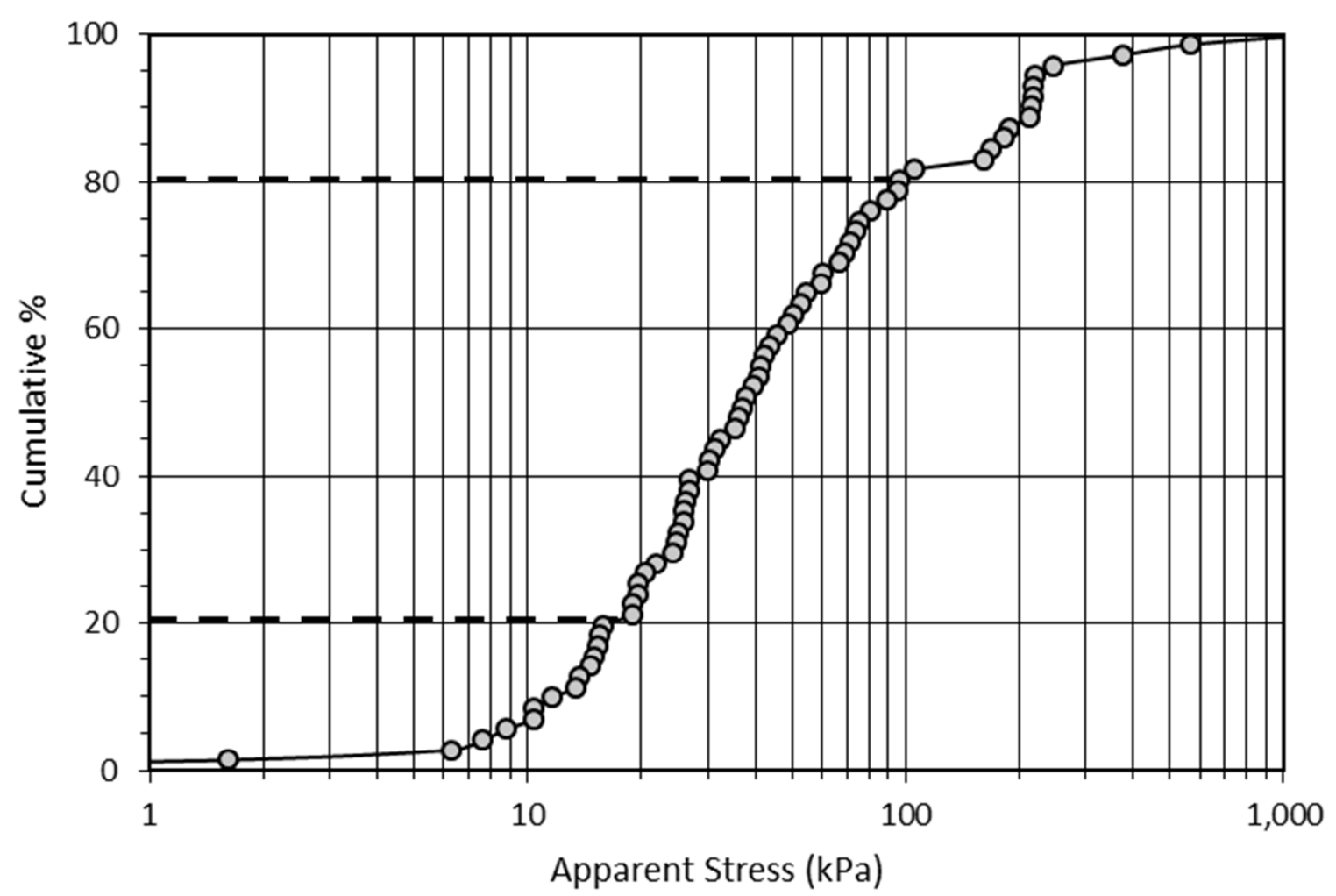

Figure 6: Cumulative distribution of apparent stress for a seismic population at a deep Canadian Mine. All events shown occur within a $30 \mathrm{~m}$ radius sphere over a time span of approximately five years. Black dashed lines approximate the extent of the linear relation for the data, corresponding to the 20th and 80th apparent stress percentiles. 


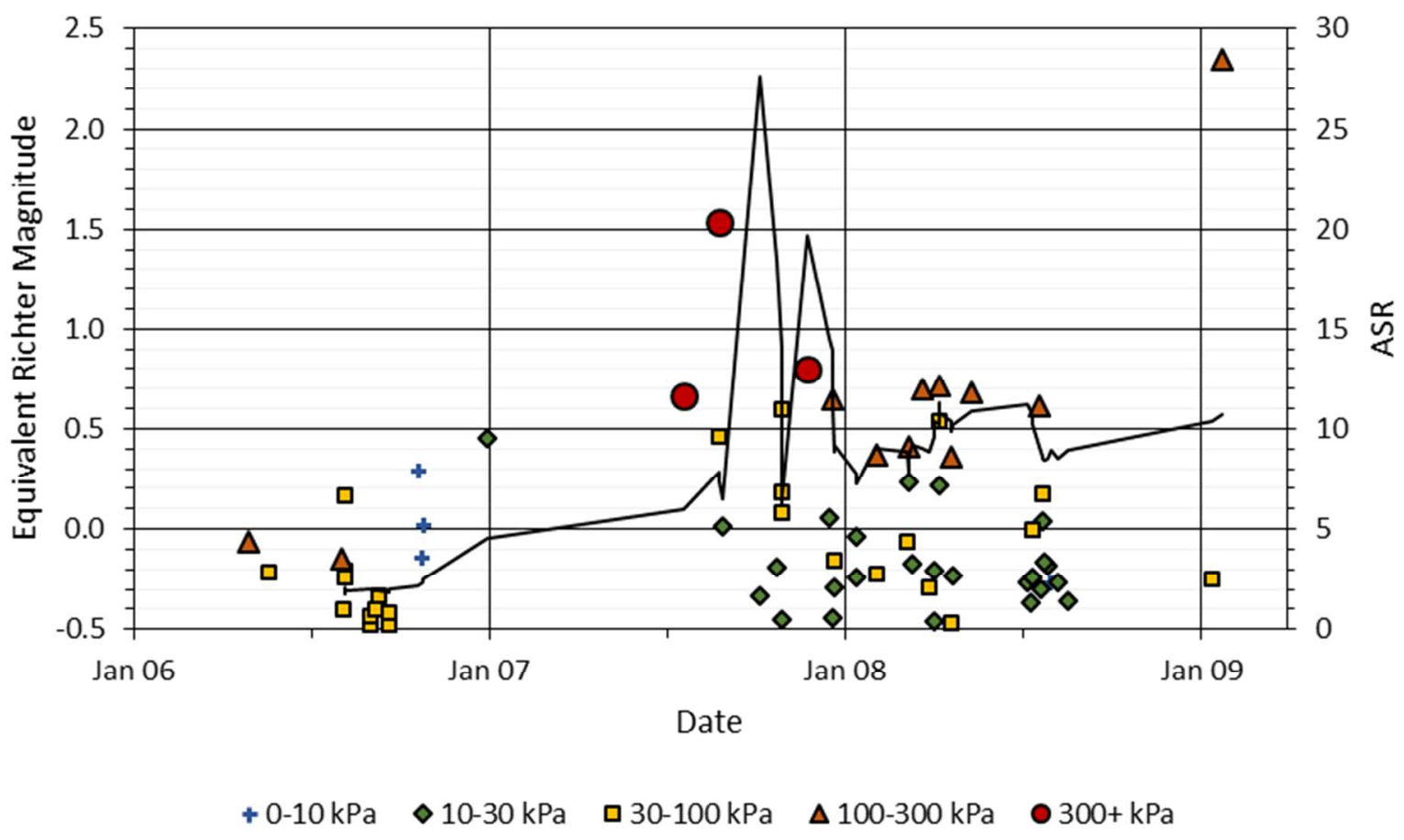

Figure 7: Apparent Stress Ratio Time History chart for a seismic population containing large magnitude events $(M R>1)$. All events shown occur within a $30 \mathrm{~m}$ radius sphere. Individual ASR values are calculated for long term seismic assessment - considering events within a one year proceeding time period. ASR increases prior to the occurrence of large magnitude seismic events. 


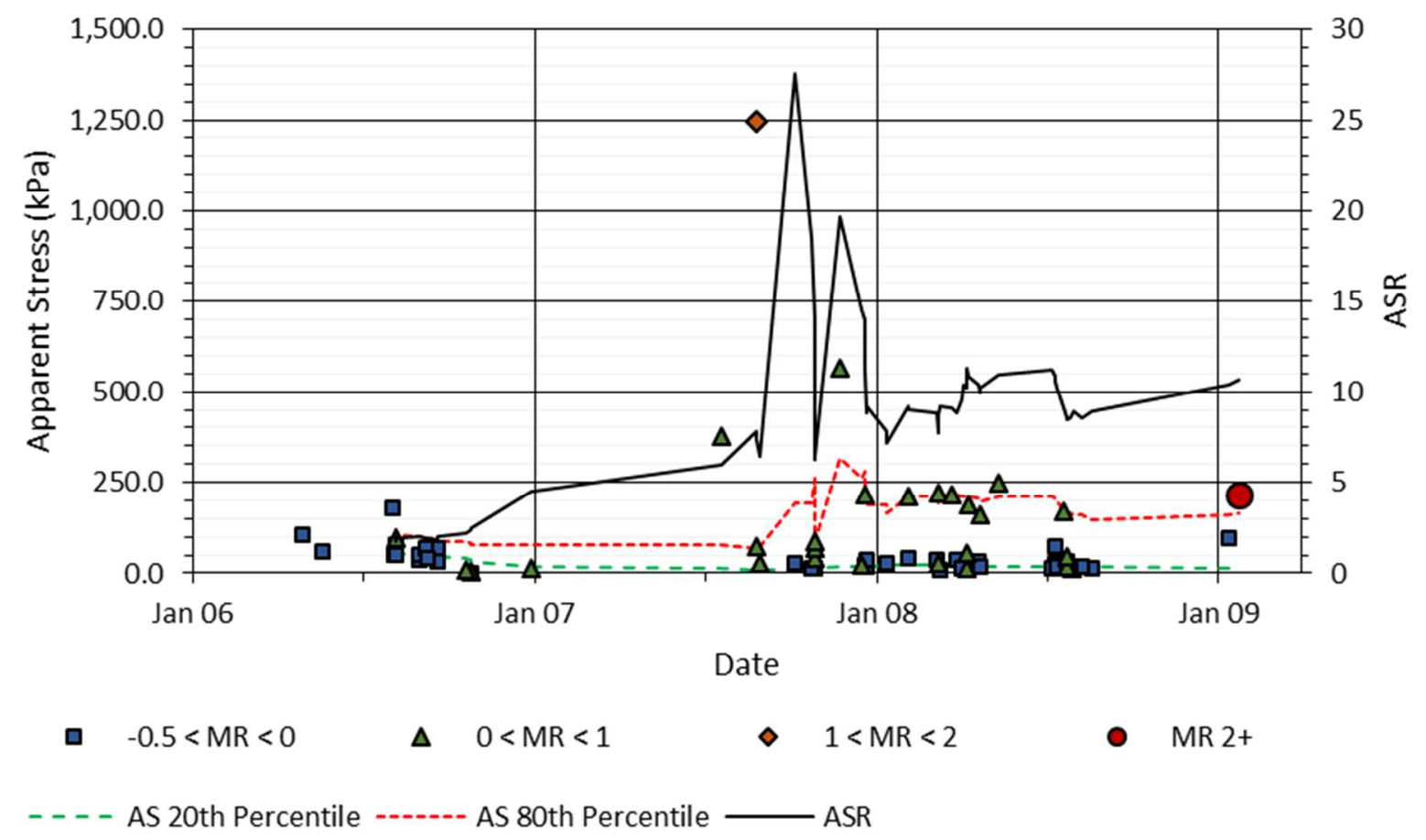

Figure 8: Apparent Stress Percentile Time History chart for a seismic population containing large magnitude events $(M R>1)$. All events shown occur within a $30 \mathrm{~m}$ radius sphere. Individual apparent stress percentile and ASR values are calculated using a 1 year trailing seismic population - considering events within a one year preceding time period. Apparent stress 20th percentile values remain relatively stable over time with increases in ASR being driven by increases in the apparent stress 80 th percentile values. 


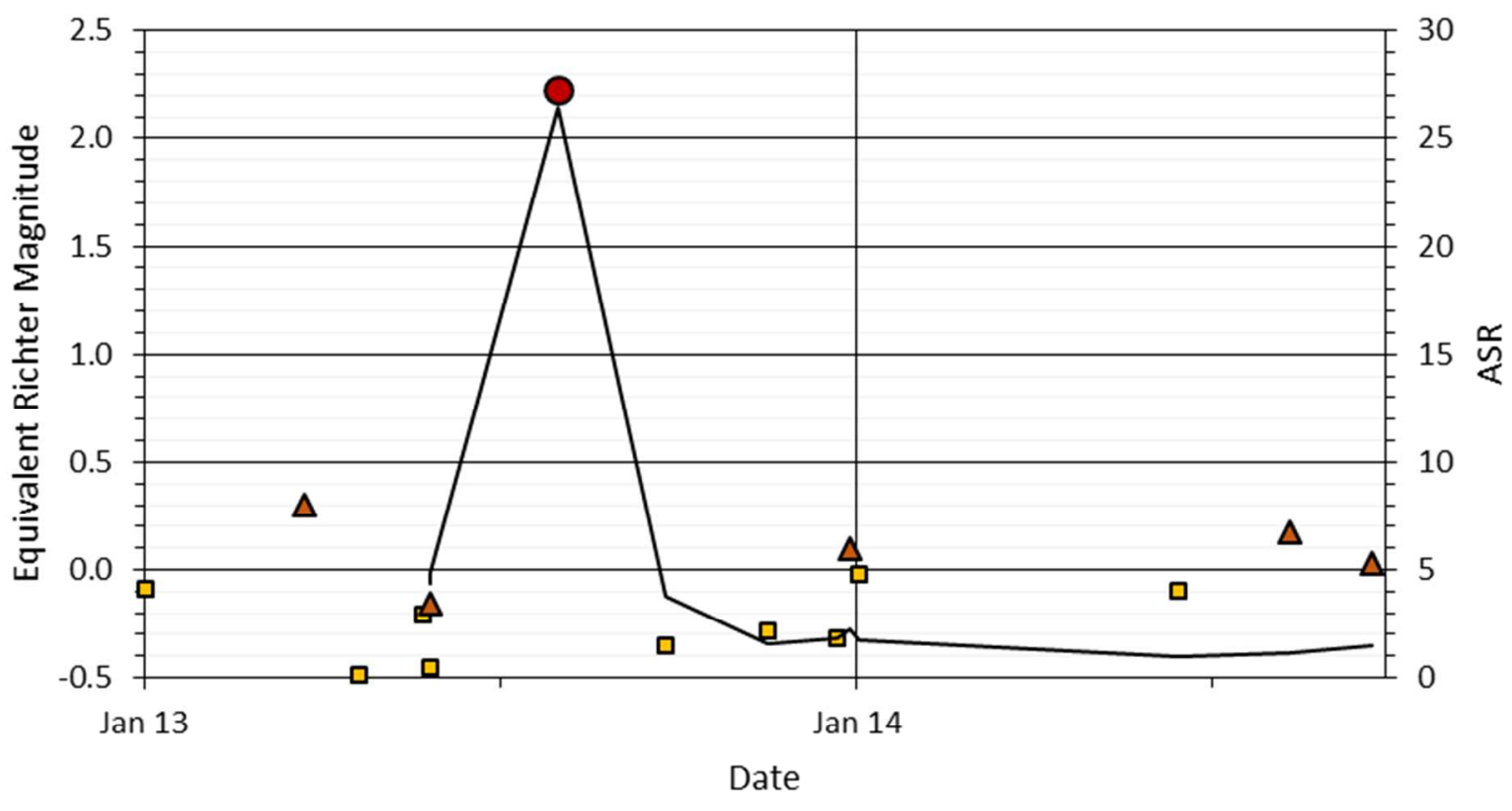

口 30-100 kPa $\quad \Delta 100-300 \mathrm{kPa} \quad 0300+\mathrm{kPa}$

Figure 9: Apparent Stress Ratio Time History chart for a seismic population containing large magnitude events $(\mathrm{MR}>1)$. All events shown occur within a $30 \mathrm{~m}$ radius sphere. Individual ASR values are calculated for medium term seismic assessment - considering events within a three month preceding time period. ASR increases prior to the occurrence of the large magnitude seismic event. 


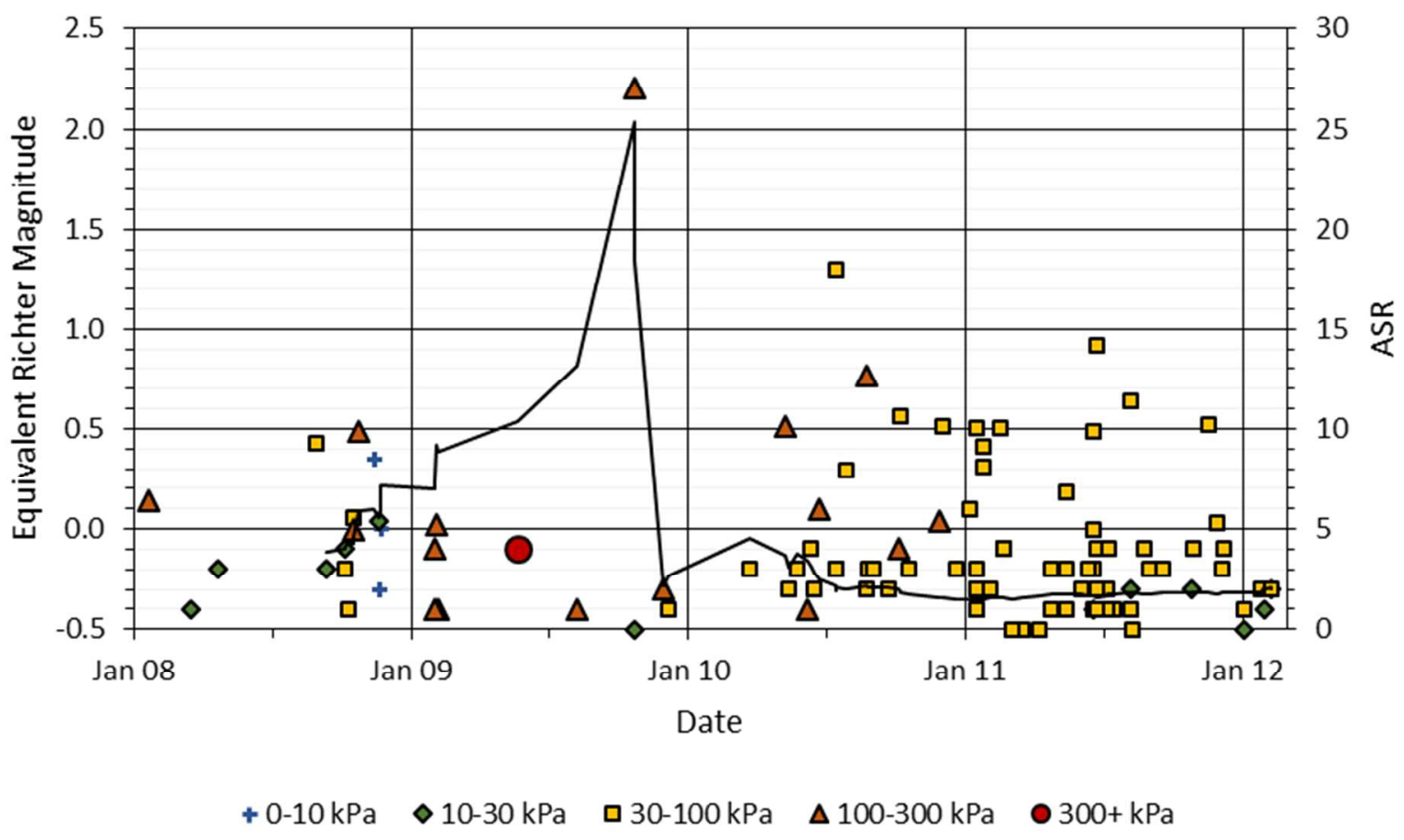

Figure 10: Apparent Stress Ratio Time History chart for a seismic population containing large magnitude events $(\mathrm{MR}>1)$. All events shown occur within a $30 \mathrm{~m}$ radius sphere. Individual ASR values are calculated for long term seismic assessment - considering events within a one year preceding time period. ASR values reflect both a local increase and decrease in rock mass stress conditions. 


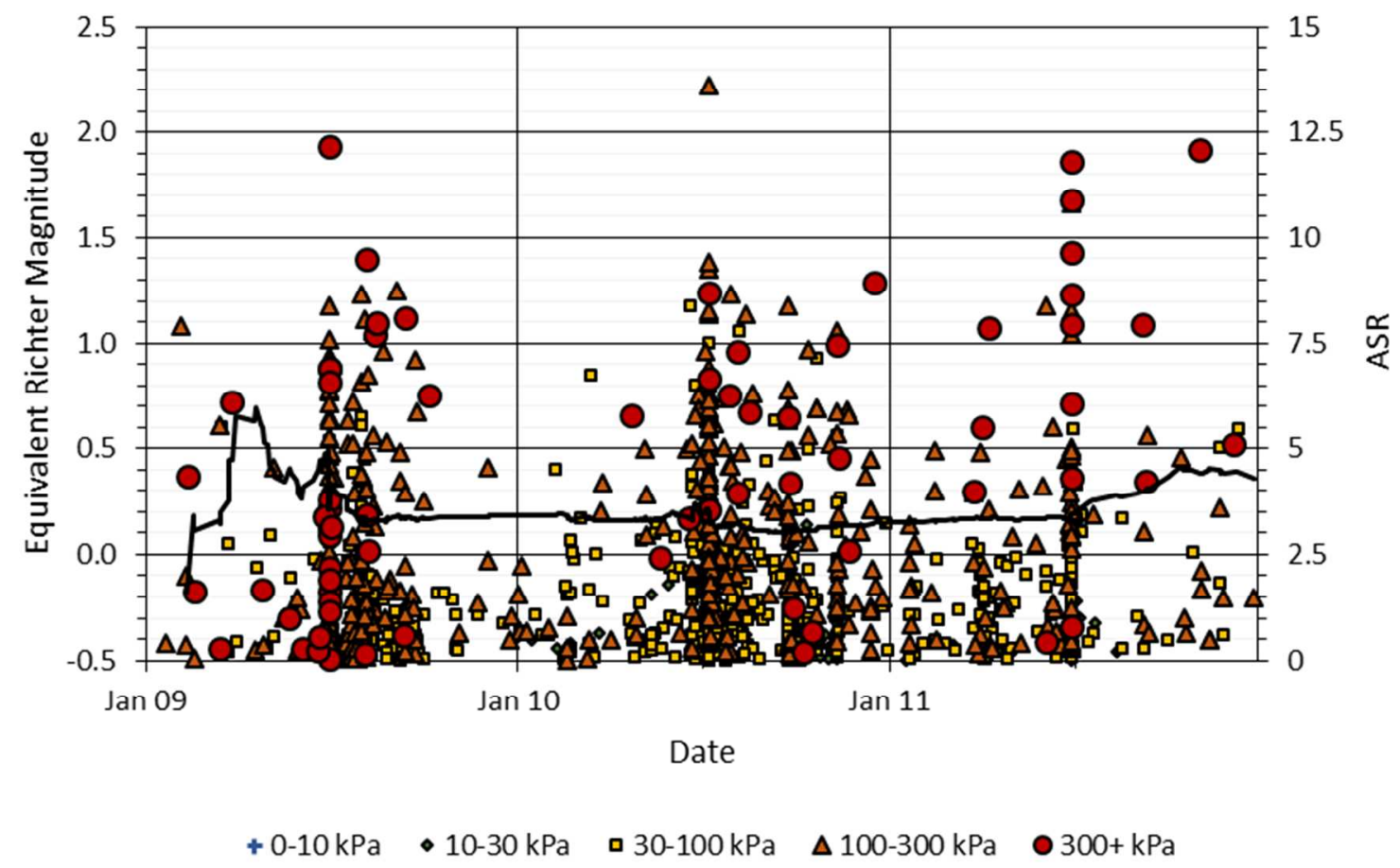

Figure 11: Apparent Stress Ratio Time History chart for a seismic population containing sequences of large magnitude events (MR $>1$ ). Individual ASR values are calculated for long term seismic assessment - considering events within a one year preceding time period. ASR values are indicative of moderate to high stress conditions throughout the population life, reflecting both stress increases and continued high rock mass stress conditions. 


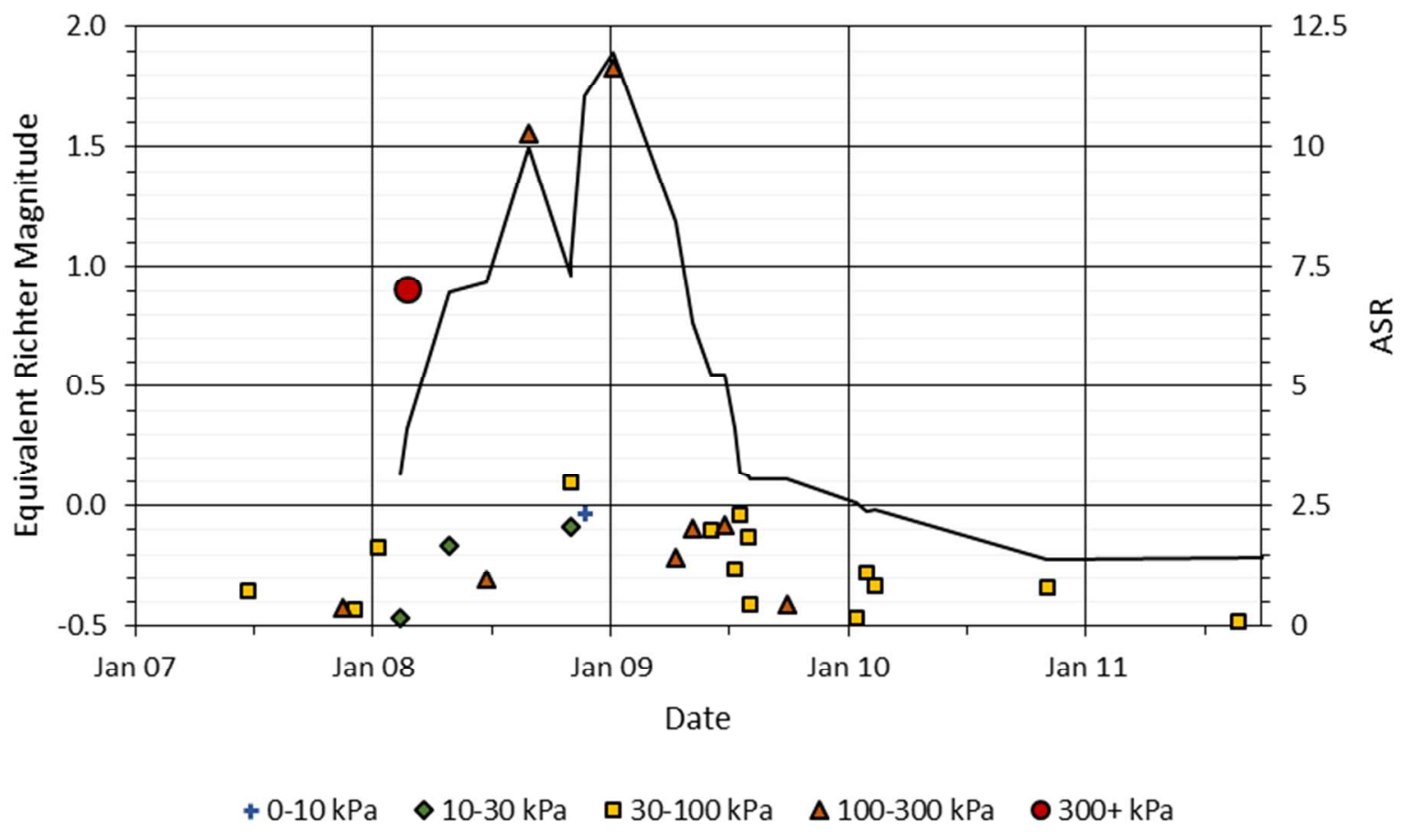

Figure 12: Apparent Stress Ratio Time History chart for a seismic population containing large magnitude events $(\mathrm{MR}>1)$. All events shown occur within a $30 \mathrm{~m}$ radius sphere. Individual ASR values are calculated for long term seismic assessment - considering events within a one year preceding time period. Peak ASR values continue to increase over time as larger magnitude events are introduced to the population. 


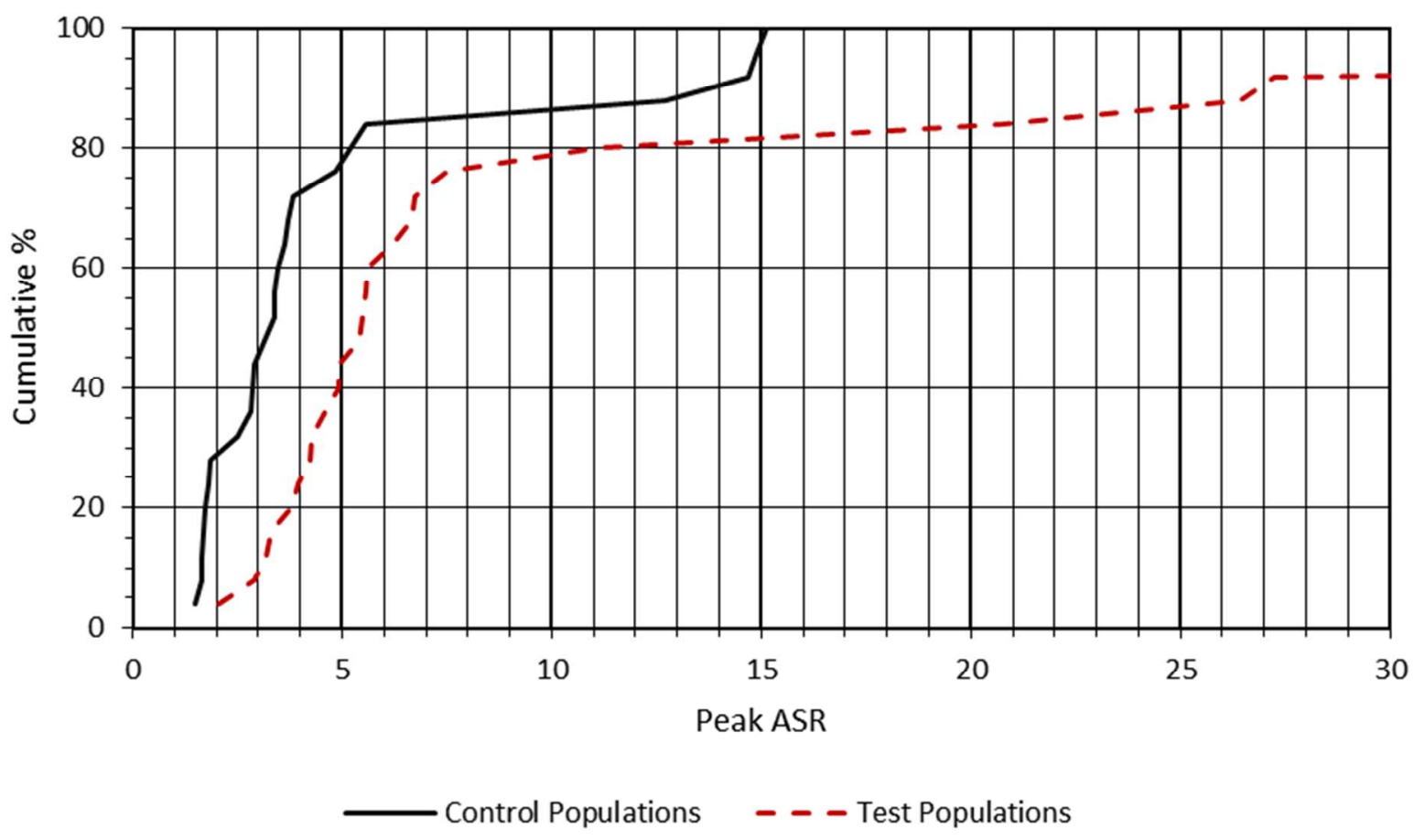

Figure 13: Cumulative distribution of peak ASR values for control and test populations calculated using medium term seismic assessment - considering events within a three month preceding time period (modified from Brown 2015). Test populations, containing large magnitude events, possess significantly larger peak ASR values. 